\title{
MicroRNA-140-5p regulates the proliferation, apoptosis and inflammation of RA FLSs by repressing STAT3
}

\author{
JIEHUA ZHU ${ }^{1}$, JIANGLIN WANG $^{2}$, JIALIN HUANG $^{2}$, WENSHENG DU ${ }^{1}$, \\ YINGZHONG HE ${ }^{1}$, HONGFEI PAN ${ }^{3}$ and JUNMIN LUO ${ }^{3}$ \\ ${ }^{1}$ Department of Laboratory Medicine, The Affiliated Hospital of Zunyi Medical University; ${ }^{2}$ School of Laboratory Medicine; \\ ${ }^{3}$ Department of Immunology, Zunyi Medical University, Zunyi, Guizhou 563000, P.R. China
}

Received September 5, 2018; Accepted April 9, 2020

DOI: $10.3892 /$ etm.2020.9602

\begin{abstract}
Ectopic expression of microRNA (miRNA) in rheumatoid arthritis (RA) fibroblast-like synoviocyte (RA FLS) is associated with the development of rheumatoid arthritis. The present study aimed to evaluate the effects of miRNA-140-5p (miR-140) on the properties of RA FLSs. It was found that miR-140 expression was decreased in 33 RA patients and extracted RA FLS samples, when compared to the corresponding healthy controls. Abnormally increased miR-140 expression in RA FLSs attenuated cell proliferation and increased cell apoptosis. Additionally, reduced pro-inflammatory cytokine production was observed in RA FLSs transfected with a miR-140 precursor. Furthermore, the 3'-UTR of the signal transducer and activator of transcription (STAT) 3 gene was identified as a target of miR-140. Notably, restoration of STAT3 expression rescued the regulatory effect of miR-140 on the proliferation, apoptosis and inflammatory cytokine production of RA FLSs. Therefore, the current findings indicated that miR-140 is a crucial modulator of both proliferation and apoptosis, shedding light on the etiology behind RA FLS viability, which is modulated by an interplay between miR-140 and STAT3 in the context of RA.
\end{abstract}

\section{Introduction}

Rheumatoid arthritis (RA) is a chronic autoimmune disease characterized by pronounced synovial hyperplasia and joint synovitis, a consequence of bone and cartilage destruction induced by the marked overproduction of inflammatory cytokines (1-3). Fibroblast-like synoviocytes (FLSs) are a critical component in the development of RA $(4,5)$ and are reportedly associated with inflammation and joint destruction in RA $(6,7)$.

Correspondence to: Dr Junmin Luo, Department of Immunology, Zunyi Medical University, 6 Xuefu West Road, Zunyi, Guizhou 563099, P.R. China

E-mail: luojunmin11@163.com

Key words: rheumatoid arthritis, fibroblast-like synoviocyte, microRNA-140, signal transducer and activator of transcription 3
Therefore, investigating the key properties of RA FLS is being increasingly applied in the search for novel therapies to block the development of RA.

MicroRNA (miRNA), a category of small, non-coding RNA, was initially identified in 1993 (8). Presently, more than thirty thousand mature miRNA sequences have been discovered in 206 species (9). There is increasing evidence to show that miRNAs have a variety of roles in many physiological and pathological processes, including in the development of RA (10). For example, Li et al (11) found a downregulation of miRNA (miR)-192 in RA, which is caused by caveolin 1 (CAV1)-mediated suppression in RA FLS. Kurowska-Stolarska et al (12) reported that miR-34a may sustain homeostatic control of $\mathrm{CD} \mathrm{c}^{+}$dendritic cells (DC) activation by regulating the expression of Axl in RA patients. Gene-silencing of miR-34a decreases the content of pro-inflammation factors (12). These investigations provide evidence in support of miRNAs as potential targets for the treatment of RA.

The Janus kinase (JAK)/signal transducer and activator of transcription (STAT) pathway is critical in the occurrence and development of RA (13-15). Specifically, the involvement of STAT3 has been reported in the progression of human malignancies (16,17), angiogenesis (18) and metastasis (19). Interactions between associated pro-inflammatory cytokines, including IL-1 $\beta$, IL-6, TNF-alpha and their receptors results in the phosphorylation of Tyr on the corresponding JAKs and the phosphorylation and dimerization of STATs. In its dimeric form, STAT is delivered to the nuclei for transcription. The JAK-STAT pathway is reportedly blocked by cytokine-signaling protein inhibitors $(20,21)$ such as CP-690550, a JAK/STAT pathway-specific inhibitor, which has demonstrated significant American College of Rheumatology 50/70 responses in phase II/III clinical trials $(22,23)$.

In the present study, the focus was on the interplay between RA and miR-140, which is widely reported to be variable depending on the specific different biological processes in various cell lines (24-33). For instance, miR-140 may influence proliferation, apoptosis and autophagy by targeting the fucosyltransferase 1 (FUT1) gene in chondrocytes during the development of osteoarthritis (25). miR-140 also has a regulatory role on proliferation, apoptosis and inflammation in non-small cell lung cancer cells (24-29), osteosarcoma 
cells (30-32) and synovial fibroblasts (33). Therefore, the present study conducted a novel investigation into the difference in expression levels of miR-140 in RA patients and their healthy counterparts. Considering the influence of miR-140 in regulating various biological process (34-36), the aim of the present study was to understand how miR-140 affects the biological events in RA FLS and to elucidate the mechanism of action behind how miR-140 regulates STAT3 expression in RA FLS.

\section{Materials and methods}

Sample origins. Synovial tissue samples were collected from 33 RA patients after knee replacement surgeries from the Affiliated Hospital of Zunyi Medical University, of which 17 were male and 16 were female, with an average age of 55.1 \pm 13.8 years and an age range of 40.1-61.0 years. RA diagnoses were made based on previous reference standards (37). Normal synovial biopsies from 8 patients with traumatic knee injuries were used as healthy controls, of which 5 were male and 3 were female, with an average age of $39( \pm 10)$ years. All study participants had given their written informed consent before participating in the study and the study was approved by the Ethics Committee of Zunyi Medical University.

Cell culture. Healthy and RA human FLSs were obtained from 3 healthy volunteer and RA patients, which were treated with $2.5 \mathrm{~g} / 1$ trypsin at $37^{\circ} \mathrm{C}$ for $2 \mathrm{~h}$. Culture of RA and healthy FLSs was carried out in DMEM (Gibco; Thermo Fisher Scientific, Inc.) supplemented with $10 \%(\mathrm{v} / \mathrm{v})$ heat-inactivated fetal bovine serum (FBS; Gibco; Thermo Fisher Scientific, Inc.), $100 \mathrm{U} / \mathrm{ml}$ penicillin (Beyotime Institute of Biotechnology) and $100 \mathrm{mg} / \mathrm{ml}$ streptomycin (Beyotime Institute of Biotechnology), followed by 3-6 passages. Cells were cultured in an incubator at $37^{\circ} \mathrm{C}, 5 \% \mathrm{CO}_{2}$ with saturated humidity. Following $48 \mathrm{~h}$ of incubation, the medium was changed, and cells were passaged when reaching $80-90 \%$ confluence.

Plasmid construction and transfection. miR-140-5p and miR-NC was obtained from Origene Technologies, Inc. The mature-miR-140-5p and miR-NC sequence was cloned into pCDHCMV-MCS-EF1-coGFP constructs (Invitrogen; Thermo Fisher Scientific, Inc.). For STAT3 overexpression, the coding sequences of STAT3 were amplified from cDNA isolated from RA FLS using a PCR kit (AP111-11; TransGen Biotech Co., Ltd.). The thermocycling conditions were as follows: $95^{\circ} \mathrm{C}$ for $10 \mathrm{~min}$, followed by 40 cycles of $95^{\circ} \mathrm{C}$ for $15 \mathrm{sec}, 60^{\circ} \mathrm{C}$ for $30 \mathrm{sec}$ and $72^{\circ} \mathrm{C}$ for $30 \mathrm{sec}$. Sequences for primers were as follows: STAT3 forward, 5'-GACTTAGTC CCAGGTACT-3' and reverse, TTCAACTGACCTAGGACG TGGTCG-3'. The product was inserted into the pcDNA3.1 vector (Invitrogen; Thermo Fisher Scientific, Inc.) to generate STAT3 expression vectors pcDNA3.1-SOX11. Cells were transfected with pCDHCMV-MCS-EF1-coGFP-miR-140-5p and/or pcDNA3.1-SOX11 using Lipofectamine ${ }^{\circledR}$ RNAiMAX/2000 transfection reagent (Invitrogen; Thermo Fisher Scientific, Inc.) and then collected at $48 \mathrm{~h}$ after transfection. All constructions were confirmed using plasmid DNA sequencing using the dideoxy chain-termination (Sanger) method with Applied Biosystems 3730XL (Genescript).
Cell Counting Kit (CCK)-8 assay. Viability of FLSs was determined using CCK-8 assays (Sigma-Aldrich; Merck KGaA) in 96-well plates. The kit was used according to the manufacturer's protocol. Cells were seeded in the 96 -well plates at a density of $5 \times 10^{3}$ cells/well and cultured until they reached $80 \%$ confluence and the aforementioned plasmids were transfected into the FLS cells. At 72, 48, 24 and $0 \mathrm{~h}$ post-transfection, CCK- 8 reagents were added to each well and incubated for $1 \mathrm{~h}$.

An independent standard curve was drawn to calculate the cell numbers. Briefly, $1 \times 10^{7}$ cells were counted using a Countess II (Thermal Fisher Scientific, Inc.) and then these cells were diluted 2 -fold and the absorbance at $450 \mathrm{~nm}$ was detected. Cell numbers in the other wells was calculated from the standard curve.

BrdU incorporation assay. After a total of $48 \mathrm{~h}$ after transfection of the FLSs, the cells were incubated for $1 \mathrm{~h}$ in BrdU stock solution, diluted 1/1,000 in PBS, followed by a wash in PBS. Cells underwent a subsequent fixation in $4 \%$ paraformaldehyde for $20 \mathrm{~min}$ at room temperature, followed by permeabilization using Triton X-100 for $10 \mathrm{~min}$ at room temperature. After subsequent blocking with $10 \% \mathrm{FBS}$ for $1 \mathrm{~h}$ at $37^{\circ} \mathrm{C}$, cells underwent overnight incubation with primary rabbit antibodies against $\operatorname{BrdU}$ (1:200; cat. no. ab152095; Abcam) at $4{ }^{\circ} \mathrm{C}$, followed by a PBS wash and incubation using the corresponding secondary antibodies bound to Cy3 (1:500, cat. no. ab6939; Abcam) for 2 h. An independent standard curve was generated to measure FLS viability according to the method discussed previously.

MTT assay. After transfection for $24 \mathrm{~h}$, cells were seeded at a density of $5 \times 10^{3}$ cells/well in the 96 -well plates and incubated for $48 \mathrm{~h}$ at $37^{\circ} \mathrm{C}$. Three replicates were used for each experimental group. MTT substrates (Promega Corporation) were then added and incubated for $4 \mathrm{~h}$ at $37^{\circ} \mathrm{C}$. The proliferative ability of cells was reflected in the absorbance at $570 \mathrm{~nm}$.

Western blotting. Cells were suspended in RIPA buffer supplemented with protease suppressor (Roche Diagnostics) for lysis and a BCA Protein Quantitation kit was used for protein quantification. 10\% SDS-PAGE was used to isolate protein specimens $(100 \mu \mathrm{g})$, which were subsequently transferred onto $0.45 \mu \mathrm{m}$ PVDF membranes. After blocking in 5\% BSA for $1 \mathrm{~h}$, proteins on the membrane were incubated with their respective primary antibodies at $4{ }^{\circ} \mathrm{C}$ overnight. The primary antibodies used were as follows: Beta-Actin (1:5,000; cat. no. ab8227; Abcam), IL-1 $\beta$ (1:1,000; cat. no. ab2105; Abcam), IL-6 (1:1,000; cat. no. ab9324; Abcam), IL-8 (1:1,000; cat. no. ab18672; Abcam), TNF-alpha (1:1,000; cat. no. ab9635; Abcam), STAT3 (1:2,000; cat. no. ab5073; Abcam), Bcl-2 (1:2,000; cat. no. ab59348; Abcam), Bax (1:5,000; cat. no. ab53154; Abcam), and cleaved caspase-3 (1:2,000; cat. no. 9664; CST Biological Reagents Co., Ltd.). Following three washes in TBS-T, proteins were probed with secondary antibodies for $1 \mathrm{~h}$ at room temperature. Analyses of protein expression levels were performed with $\beta$-Actin an internal reference using the SuperSignal West Femto Maximum Sensitivity Substrate kit (Thermo Fisher Scientific, Inc.) and a C-DiGit Blot Scanner and Analyser (LI-COR Biosciences). 

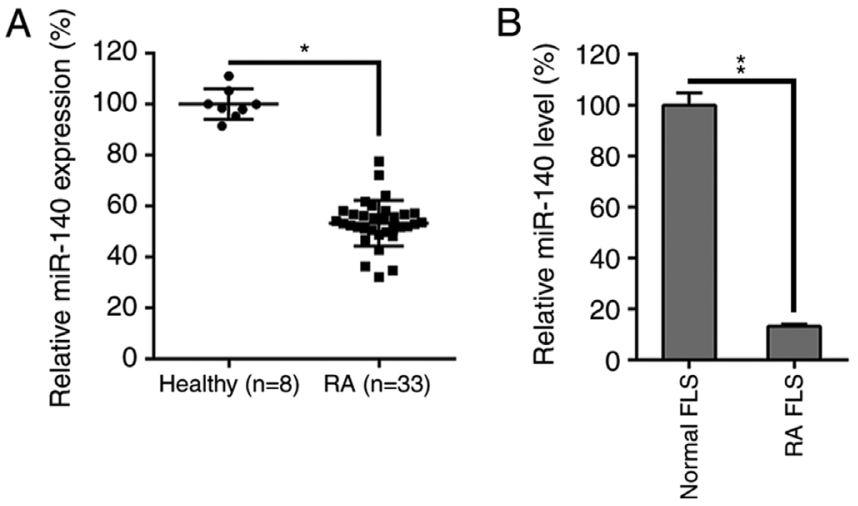

Figure 1. Expression of miR-140 in the synovial tissue of patients with RA patients and in FLSs. (A) Reduced expression levels of miR-140 were found in the synovial tissue of RA patients $(n=33)$ versus that of healthy synovial tissue ( $n=8$ ), as found through RT-qPCR. (B) Decreased expression levels of miR-140 were observed in RA FLSs as found through RT-qPCR, compare to that of healthy FLSs. Data is shown as the mean \pm SD. ${ }^{*} \mathrm{P}<0.05,{ }^{* *} \mathrm{P}<0.01$ FLS, fibroblast-like synoviocyte; miR, microRNA; RA, rheumatoid arthritis; RT-qPCR, reverse transcription-quantitative PCR.

$R N A$ extraction and reverse transcription-quantitative $P C R$ $(R T-q P C R)$. Trizol ${ }^{\circledR}$ (Thermo Fisher Scientific, Inc.) was used to isolate total RNA from FLSs. First-strand cDNA was synthesized using total RNA with a PrimeScript RT Master Mix (Takara Bio, Inc.). The samples were initially incubated at $25^{\circ} \mathrm{C}$ for $10 \mathrm{~min}$, then subsequently incubated at $42^{\circ} \mathrm{C}$ for $1 \mathrm{~h}$. SYBR-Green was incorporated to evaluate transcription and GAPDH used as internal reference for the various genes evaluated.

Quantitative PCR was conducted in $20 \mu \mathrm{l}$ volumes, with the following temperature protocol: $95^{\circ} \mathrm{C}$ for $10 \mathrm{~min}$, followed by 40 cycles of $95^{\circ} \mathrm{C}$ for $15 \mathrm{sec}, 60^{\circ} \mathrm{C}$ for $30 \mathrm{sec}$, and $72^{\circ} \mathrm{C}$ for $30 \mathrm{sec}$. Quantification was performed using the $2^{-\Delta \Delta \mathrm{Cq}}$ method (38), normalizing to GAPDH. The primer sequences used for detected genes were: miR-140 forward, 5'-TGCGGC AGTGGTTTTACCCTATG-3' and reverse, 5'-CCAGTGCAG GGTCCGAGGT-3'; IL-1 $\beta$ forward, 5'-TCAGGCAGATGG TGTCTGTC-3' and reverse, 5'-GGTCTATATCCTCCAGCT GC-3'; IL-6 forward, 5'-AACGCCTGGAAGAAGATGCC-3' and reverse, 5'-CTCAGGCTGAACTGCAGGAA-3'; IL-8 forward, 5'-GAAGATAGATTGCACCGATG-3' and reverse, 5'-CATAGCCTCTCACACATTTC-3'; TNF-alpha forward, 5'-GCTGTACCTCATCTACTCCC-3' and reverse, 5'-TAG ACCTGCCCAGATTCAGC-3'; STAT3 forward, 5'-GGAACA AGCCCCAACCGG-3' and reverse, 5'-CTAAAATCAGGG GTCCCAACTG-3'; Bcl-2 forward, 5'-CATTTCCACGTC AACAGAATTG-3' and reverse, 5'-AGCACAGGATTGGAT ATTCCAT-3'; Bax forward, 5'-AGCTGAGCGAGTGTCTCA AG-3' and reverse 5'-GTCCAATGTCCAGCCCATGA-3'; U6 forward, 5'CCTTATGCAGTTGCTCTCC-3' and reverse, 5'-CAGGAAACAGCTATGAC-3'); and GAPDH forward, 5'-GCTCAGACACCATGGGGAAGGT-3' and reverse 5'-GTGGTGCAGGAGGCATTGCTGA-3'.

Hoechst 33342 staining. FLSs at a density of $1 \times 10^{5}$ cells $/ \mathrm{ml}$ were inoculated in 12 -well plates prior to transfection. Following $48 \mathrm{~h}$ post-transfection, washing in PBS was carried out in triplicates, followed by fixing with
$4 \%$ paraformaldehyde for $15 \mathrm{~min}$ at room temperature and incubation with $500 \mu 1$ Hoechst 33342 solution for $30 \mathrm{~min}$ at $37^{\circ} \mathrm{C}$ in the dark. A fluorescence microscope (Olympus Corporation) was used to observe DNA staining. Ten fields were randomly selected and a total of 200 cells were counted. The following formula was used to calculate percentages of positively stained cells: Apoptotic cells/the total number of counted cells.

Annexin V-FITC/PI analysis. FLSs were harvested at $48 \mathrm{~h}$ post-transfection. An annexin V-FITC/propidium iodide (PI) apoptosis detection kit (Abcam) was used to detect cell apoptosis according to the manufacturer's guidelines. Beckman Coulter FACSCalibur (version 6.0, Beckman Coulter, Inc.) was used to calculate the percentage of apoptotic cells, using flow cytometry. Flow cytometry density plots showing annexin $\mathrm{V}$ (X-axis) and PI (Y-axis) staining of cells were generated using FACStation software (version 6.0; BD Bioscience). The right lower quadrant represents annexin $\mathrm{V}$ positive/PI negative staining, which indicates early apoptosis. The right upper quadrant represents both high annexin V and PI staining, indicating late apoptosis and the left upper quadrant represents low annexin V and high PI staining, indicating necrosis. The left lower quadrant indicates viable cells.

In vitro caspase-3 activity assay. Caspase-3 activity was determined using colorimetric assay kits, which utilized synthetic tetrapeptides [Asp-Glu-Val-Asp (DEAD) for caspase-3] (cat. no. A1086; Sigma-Aldrich; Merck KGaA) labeled with p-nitroaniline (pNA; Abcam; cat. no. ab39401). The kits were used according to the manufacturer's protocols. Briefly, cells were lysed in the supplied lysis buffer for $30 \mathrm{~min}$ at $4^{\circ} \mathrm{C}$. Supernatants were collected and incubated with the supplied reaction buffer containing dithiothreitol and DEAD-pNA as substrates at $37^{\circ} \mathrm{C}$. The reactions were measured by changes in absorbance at $405 \mathrm{~nm}$ using the VERSAmax tunable microplate reader.

Dual-luciferase reporter assay. The STAT3 gene was used as a target and amplification of the STAT3 gene 3'-UTR was carried out, followed by fusion in psiCHECK-2 vector (Promega Corporation) with the GV126 luciferase gene (Promega Corporation). The binding sites of the STAT3 gene and miR-140 were eliminated by site-directed mutation, producing a mutant which was used as a control. Thymidine kinase promoters (pRL-TK vectors; Takara Bio, Inc.) and the plasmid containing Renilla luciferase were used to adjust for transfection efficiency. Co-transfection of control and miR-140 with luciferase reporter vectors into RA FLSs was carried out using Lipofectamine 2000 (Thermo Fisher Scientific, Inc.). At $36 \mathrm{~h}$ post transfection, the cells were cultured in myllicin-containing DMEM, which was supplemented with $10 \%$ FBS (Gibco; Thermo Fisher Scientific, Inc.) and cells then placed into an incubator with $5 \%$ carbon dioxide at $37^{\circ} \mathrm{C}$. The liquid was replaced every $48 \mathrm{~h}$. After treatment with trypsin, cells were subcultured until they reached $80 \%$ confluency. The relative light unit of the firefly luciferase was recorded with a GloMax 96 microplate luminometer (Promega Corporation). The ratio of firefly to Renilla was used to normalize the firefly luciferase values. 
B

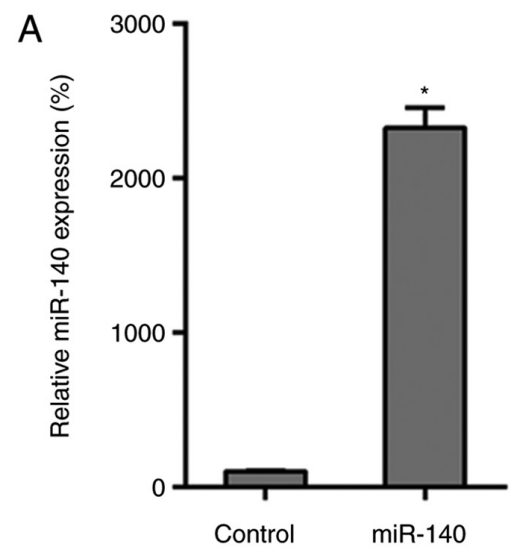

C

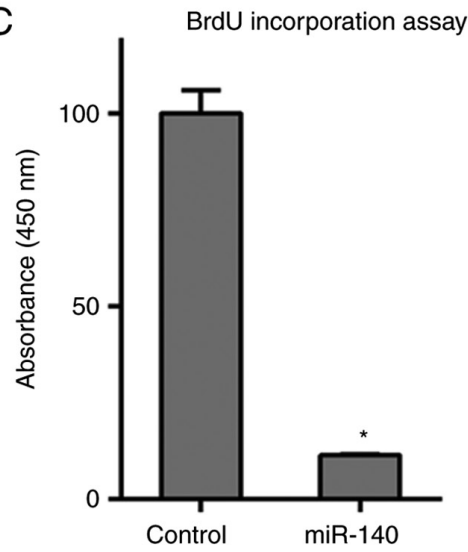

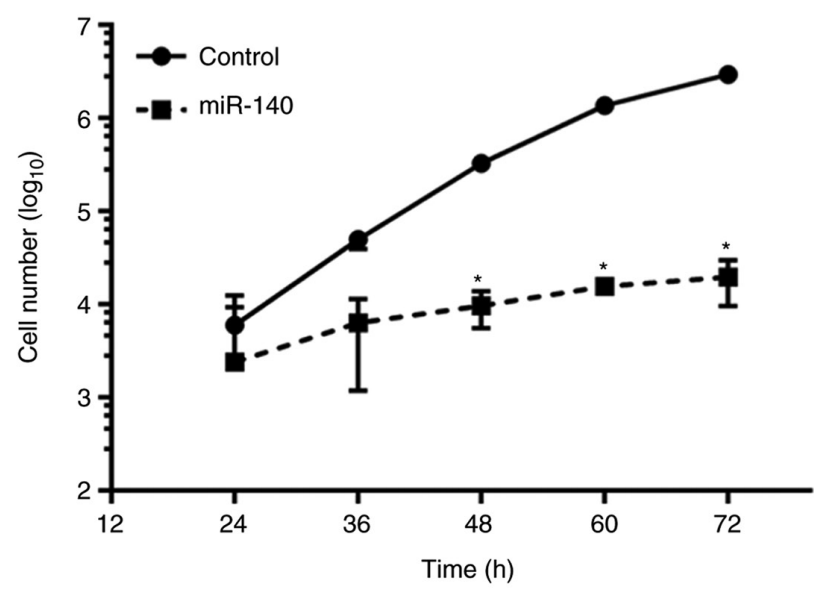

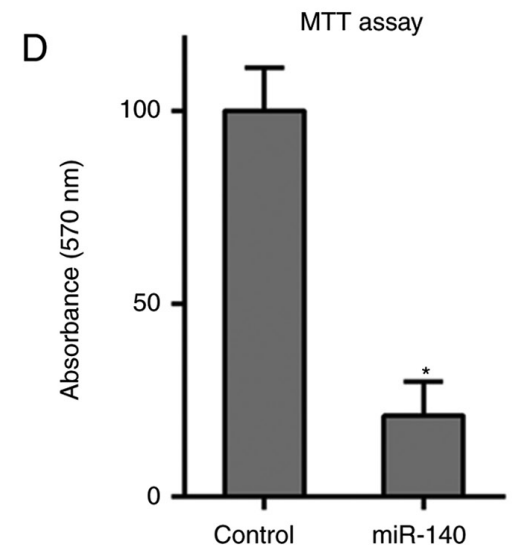

Figure 2. miR-140 overexpression inhibited the proliferation of RA FLSs. (A) RT-qPCR assays were carried out in order to evaluate miR-140 expression levels in RA FLSs following transfection with miR-140 precursor or miR-NC. All groups were normalized to the miR-NC group (100\%). (B) CCK-8 assays were used to examine cell viability of RA FLSs at various time points. (C) BrdU incorporation assay was used to examine cell survival at $48 \mathrm{~h}$ post-transfection. (D) MTT assays were performed to evaluate the cell proliferative ability at $48 \mathrm{~h}$ post-transfection. Outcomes are presented as the mean $\pm \mathrm{SD}$. ${ }^{*} \mathrm{P}<0.05$ vs. the corresponding control. CCK, Cell Counting Kit; FLS, fibroblast-like synoviocyte; miR, microRNA; RA, rheumatoid arthritis.

TargetScan prediction. The prediction algorithm database, TargetScan (www.targetscan.org) was used to nominate targets of miR-140. Using the database, predictions were ranked based on the predicted efficacy of targeting as calculated using the cumulative weighted context ++ scores of the sites (39). As an option, predictions are also ranked by their probability of conserved targeting (40).

Statistical method. Data are expressed as the mean \pm SD. Intergroup distinctions were evaluated using one-way ANOVAs with Tukey's post hoc tests, or two-tailed Student's t-tests. $\mathrm{P}<0.05$ was considered to indicate a statistically significant difference.

\section{Results}

miR-140 is downregulated in the synovial tissue of $R A$ patients and RA FLSs. To evaluate how miR-140 was influenced by RA progression and FLS functions, the miR-140 expression levels in RA patients and isolated FLS lines were measured. miR-140 expression was first evaluated in $33 \mathrm{RA}$ specimens and 8 healthy specimens. A decrease was identified in the miR-140 levels in the RA samples compared to healthy controls (Fig. 1A). Furthermore, miR-140 expression levels were also remarkably lower in the RA FLSs compared to healthy controls (Fig. 1B).

Overexpression of miR-140 impairs proliferation of RA FLSs. To assess how miR-140 affects RA FLS growth and proliferation, miR-140 expression was restored through transfections. This upregulation of miR-140 was first confirmed in RA FLSs after transfection with the miR-140 mimics, compared to that in miR-NC-treated cells, as found by RT-qPCR (Fig. 2A). Transfection and rescue experiments with miR-140 demonstrated a decreased cell growth rate in the RA FLSs at $48-72 \mathrm{~h}$ post-transfection compared to that in the NC group (Fig. 2B). BrdU immunofluorescence assay and MTT assays were also performed to measure cell proliferation at $48 \mathrm{~h}$ post-transfection. BrdU staining revealed that, compared with that of the control group, miR-140 transfections inhibited cell DNA synthesis in RA FLSs by approximately $80 \%$ (Fig. 2C) and results from the MTT assay further supported this result, with miR-140 transfection suppressing the cell proliferation of RA FLSs (Fig. 2D). Overall, the overexpression of miR-140 had an inhibitory effect on these FLS cells.

miR-140 overexpression caused apoptosis of RA FLSs. The miR-140-expressing cells showed that in comparison with the 
A
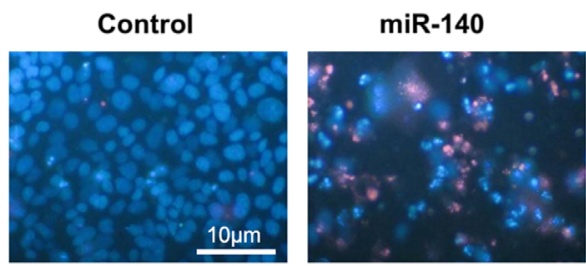

B
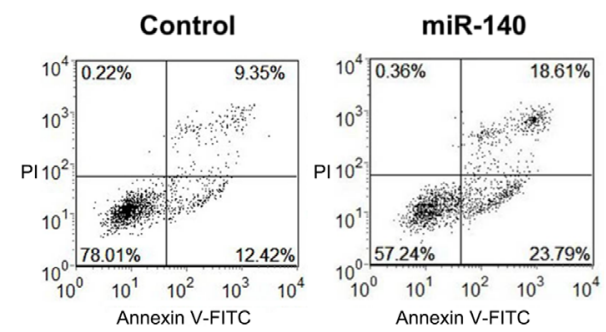

C

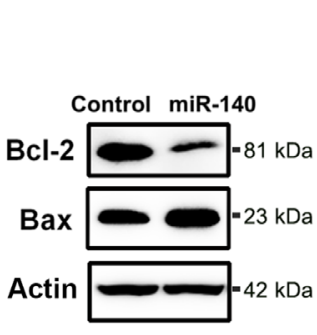

$\mathbf{F}$

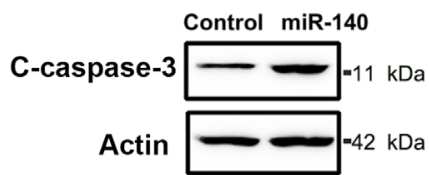

Hoechst 33342 staining

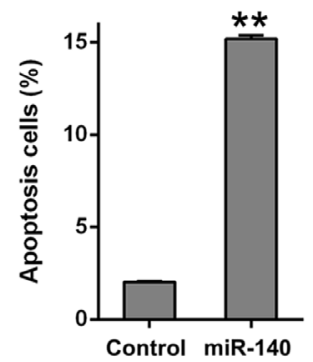

Annexin V-FITC/PI analysis

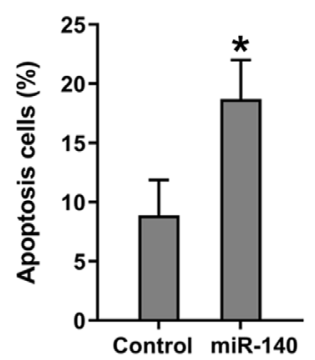

E

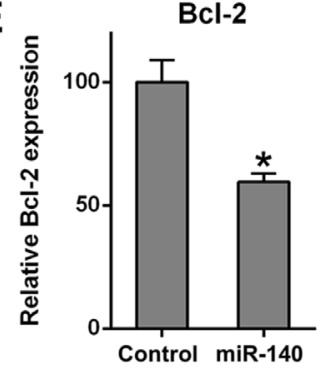

Figure 3. Ectopic overexpression of miR-140 enhances apoptosis of RA FLSs. (A) Hoechst 33342 staining was carried out on the miR-140 overexpressing or control RA FLSs. Magnification x200. The apoptotic rate of HepG2 cells, those stained positive with Hoechst 33342, are displayed in the right panel. (B) Annexin V-FITC/propidium iodide staining and flow cytometry were performed to evaluate the number of apoptotic cells. Early apoptotic cells and later apoptotic cells are indicated in the top right and bottom right quadrants, respectively, in each plot. Analysis of the apoptotic rate of RA FLSs in the two groups is displayed in the right panel. (C) Western blotting for Bcl2 and Bax, as well as reverse transcription-quantitative PCR for (D) Bax and (E) Bcl2 were performed to assess the effects of miR-140 on the Bcl-2 and Bax expression levels at the protein and mRNA levels. (F) Western blotting was carried out to examine cCaspase-3 levels following miR-140 transfection. $(\mathrm{G})$ miR-140 reduced caspase-3 activity in RA FLSs. All groups were normalized to the control group (100\%). Data represents the mean $\pm \mathrm{SD} .{ }^{*} \mathrm{P}<0.05,{ }^{* * *} \mathrm{P}<0.01$ vs. the control group. Cleaved caspase-3, cCaspase-3; FLS, fibroblast-like synoviocyte; miR, microRNA; PI, propidium iodide; RA, rheumatoid arthritis.

control, the number of positive Hoechst 33342-stained cells was higher at $48 \mathrm{~h}$ after transfection (Fig. 3A). Moreover, the flow cytometry results demonstrated that overexpression of miR-140 caused increased apoptotic activity in RA FLSs (Fig. 3B). Since miR-140 suppressed cell viability and promoted RA FLS apoptosis, its role in regulating the expression of apoptosis-related proteins was further investigated. Bcl-2 and Bax are typical anti- and pro-apoptotic proteins, respectively. In Fig. 3C-E, transfection of RA FLSs with miR-140 caused a decrease in the expression levels of Bcl-2, but increased Bax expression levels, compared with observations from the control group, at both the protein and mRNA levels. The cleavage of caspase-3, a typical apoptotic marker (41), and its activity in RA FLSs were examined to identify the role of miR-140 in apoptosis. The data revealed that miR-140 could induce the expression of cleaved caspase- 3 and facilitated the pro-apoptotic activity at 3 days post-transfection (Fig. 3F and G). These findings pointed to a 

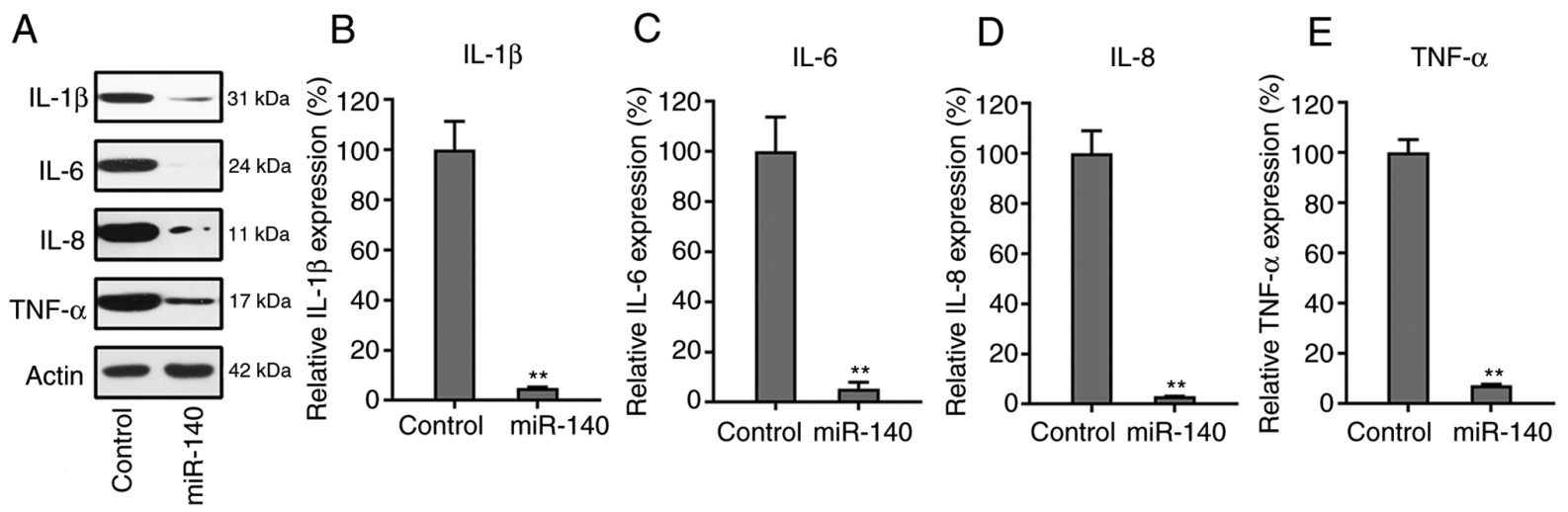

Figure 4. miR-140 represses pro-inflammatory cytokine production in the RA FLSs. (A) The protein expression of pro-inflammatory cytokines IL-1 $\beta$, IL-6, IL-8 and TNF- $\alpha$ in RA FLSs were examined using western blotting. The mRNA expression of levels (B) IL-1 $\beta$, (C) IL-6, (D) IL-8 and (E) TNF- $\alpha$ these cytokines was also determined using RT-qPCR. All groups were normalized to the control group (100\%). Data represents the mean \pm SD. ${ }^{* *} \mathrm{P}<0.01 \mathrm{vs}$. the control group. FLS, fibroblast-like synoviocyte; IL, interleukin; miR, microRNA; RA, rheumatoid arthritis; TNF, tumor necrosis factor.

mechanism of action by which miR-140 regulated apoptosis, further confirming the previous findings.

miR-140 suppresses the production of pro-inflammatory cytokines. Western blotting and RT-qPCR were used to examine the expression levels of each cytokine in the control and miR-140 overexpression groups. Notably, it was observed that the inflammatory cytokines in the miR-140-expressing cells were clearly decreased after transfection, compared to the control group (Fig. 4A). The RT-qPCR data also showed that the mRNA expression levels of the four cytokines significantly decreased when RA FLSs were transfected with the miR-140 precursor (Fig. 4B-E). These results indicated that miR-140 inhibited the production of cytokines during the development of RA.

STAT3 is a target of miR-140. Bioinformatics analysis revealed that miR-140 directly targeted STAT3, a key mediator of the JAK/STAT signaling pathway (Fig. 5A). The interaction between STAT3 and miR-140 was assessed using a dual-luciferase reporter assay. The data revealed that in miR-140-transfected RA FLSs, PTEN 3'-UTR-fused luciferase activity was reduced by $50 \%$ compared with that of the control groups (Fig. 5B). Expression of STAT3 was also examined in RA FLSs transfected with miR-140 and miR-NC using western blotting and RT-qPCR. The results conclusively showed that expression levels of STAT3 were suppressed due to miR-140 overexpression (Fig. 5C and D). The mRNA levels of STAT3 in both the normal FLSs and RA FLSs were also studied and it was found that STAT3 in RA FLSs was significantly higher than that in normal FLSs (Fig. 5E), also indicating the potential of miR-140 in regulating RA FLS properties.

Overexpression of STAT3 reverses the effect of $m i R-140$ on RA FLSs. To further determine whether STAT3 restoration rescued the regulatory effect of miR-140 on RA FLS properties, STAT3 expression was rescued in RA FLSs expressing miR-140 through transfections with a STAT3-expressing vector. First, the successful expression of STAT3 was confirmed through the transfection of STAT3-expressing vector into the cells (Fig. 6). Subsequently, STAT3 was found to be overexpressed in RA FLSs co-expressing miR-140 at the protein and mRNA levels (Fig. 7A and B). BrdU and MTT assays showed that enhanced expression of STAT3 restored the proliferation of RA FLS, previously shown to be inhibited by miR-140 (Fig. 7C and D). The relationship between the apoptotic activity of miR-140-expressing RA FLSs and STAT3 restoration was determined using annexin V-FITC/PI flow cytometry. Cell apoptosis was evidently reduced in the STAT3- and miR-140-expressing RA FLSs, compared with that of cells which were overexpressing miR-140 alone and with that of the control cells (Fig. 7E). Furthermore, western blotting and RT-qPCR were used to investigate signs of inflammation of RA FLSs expressing both STAT3 and miR-140. Overexpression of STAT3 was observed to also cause a reverse effect on the downregulation of inflammatory cytokines at the protein and mRNA level, which was mediated by miR-140 transfection (Fig. 7F). Taken together, restoration of STAT3 expression may recover the growth and apoptotic abilities, as well as restore the expression of inflammatory cytokine production, of RA FLSs disrupted by miR-140.

\section{Discussion}

Increasing evidence has demonstrated that miRNAs are closely related to RA progression (16). It has been reported that many miRNAs such as miR-30a (42), miR-155 (43), miR-223 (44) and miR-27a (45) are involved in modulating RA occurrence and development. Hence, miRNAs have been posited as a potential therapeutic strategy for RA treatment (10). For instance, it has been reported that involvement of miR-21 facilitates the proliferation of FLSs in RA models through $\mathrm{NF}-\kappa \mathrm{B}$ pathway (46). However, the relevant mechanism and dysfunction of miR-140-5p in RA is not well established.

Accumulating miRNAs exert their biological roles by regulating their target molecules. miR-140 has been documented to target different genes, such as AKT3 (47), Smad3 (48) and E2F8 (49), which are all involved in different biological process. In the present study, using bioinformatic analysis, the target of miR-140 and the conservative site of STAT3 were predicted. From the dual-luciferase reporter 

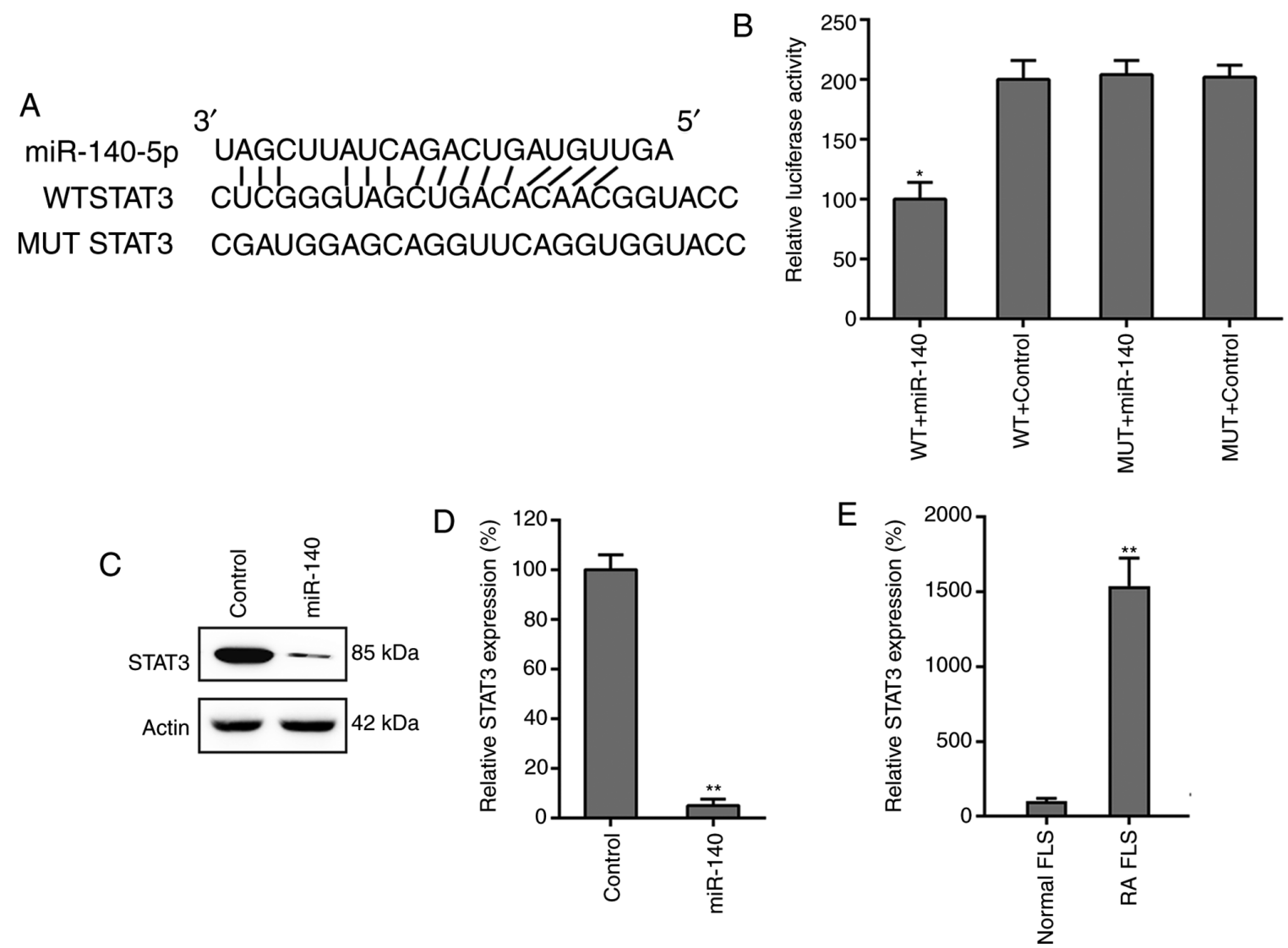

Figure 5. STAT3 is a direct target of miR-140. (A) Graphical representation of the conserved miR-140 binding motif at the STAT3 3'-UTR. The complementary sequences to the seed regions of miR-140 and corresponding sequence of the 3'-UTR of STAT3. (B) The luciferase activity exhibited by the reporter constructs, containing either the WT or MUT human STAT3 3'-UTR after miR-140 transfection. The observed luciferase activity was normalized to that of $\beta$-galactosidase. Overexpression of miR-140 markedly decreased the relative luciferase activity in the WT 3'-UTR, but not the MUT 3'-UTR, of STAT3 mRNA. All groups were normalized to the WT + control group (100\%). (C) Western blotting and (D) RT-qPCR analysis were used to evaluate the STAT3 protein and mRNA expression levels after transfection of RA FLSs with miR-140 or miR-negative control. All groups were normalized to the control group (100\%). (E) Increased expression levels of STAT3 in RA FLSs was observed by RT-qPCR, compared to that of healthy FLSs. All groups were normalized to the normal FLS group (100\%). Data represents the mean $\pm \mathrm{SD} .{ }^{*} \mathrm{P}<0.05,{ }^{* *} \mathrm{P}<0.01$ vs. the corresponding control. FLS, fibroblast-like synoviocyte; miR, microRNA; MUT, mutant; RA, rheumatoid arthritis; RT-qPCR, reverse transcription-quantitative PCR; UTR, untranslated region; WT, wild-type.

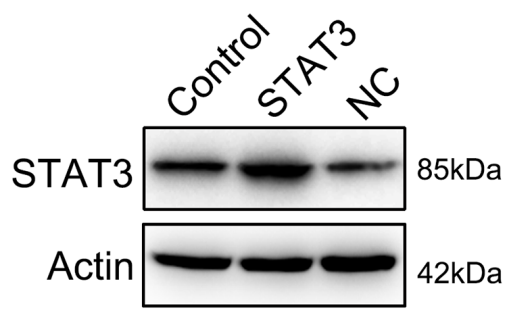

Figure 6. STAT3 was overexpressed in cells. Cells were transfected with or without STAT3-expressing vector or empty vector. Overexpression of STAT3 in the cells was then detected using western blotting at $48 \mathrm{~h}$ post-transfection. $\mathrm{NC}$, negative control.

assay, results showed that STAT3 directly interacted with miR-140 in RA FLS. STAT3 is correlated with multiple biological processes $(50,51)$, with phosphorylated STAT3 modulating various genes, including Bcl-xL, Bcl-2, VEGF and MMPs (50-53). For colorectal cancer cells, inhibition of STAT3 signaling induces apoptosis, cell cycle arrest and reduces tumor cell invasion (54). In human liver cancer cells, inhibition of STAT3 signaling blocks the anti-apoptotic activity of IL-6 (55). Inhibition of STAT3 signaling induces apoptosis and decreases survivin expression in primary effusion lymphoma (56). These reports provide a rationale for explaining the findings of the current study. Here, STAT3 was found to be decreased after aberrant expression of miR-140, and restoration of STAT3 expression in RA FLS restored the properties of FLS which were modified by miR-140, especially in reducing the apoptotic rate. This evidence suggested an important role of miR-140 in RA FLS, which is mediated by STAT3.

Since a lower apoptosis rate is one of the essential characteristics of FLS during RA development (57), a higher apoptotic rate is required to ameliorate RA progression. In the present study, overexpression of miR-140 showed a similar restorative effect by upregulating caspase- 3 and Bax, and downregulating the $\mathrm{Bcl}-2$, ultimately promoting apoptosis in RA FLS. miR-140 has been shown to exhibit its regulatory role in chondrocytes (25), cardiomyocytes (58) and ovarian cancer cells (41). Therefore, the current data suggested that miR-140 facilitates RA FLS apoptosis by both the mitochondria pathway (through reduced Bcl-2) and the death receptor pathway (through increased caspase-3).

In conclusion, this present study demonstrated that miR-140 can suppress proliferation, promote apoptosis and suppress 


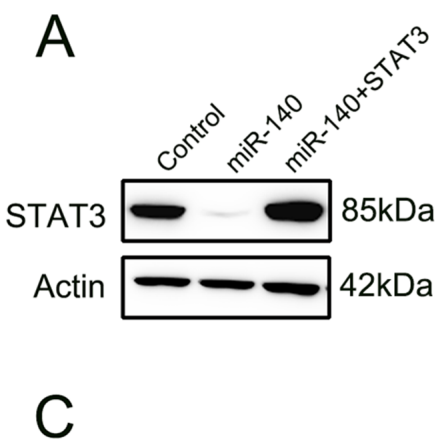

BrdU incorporation assay
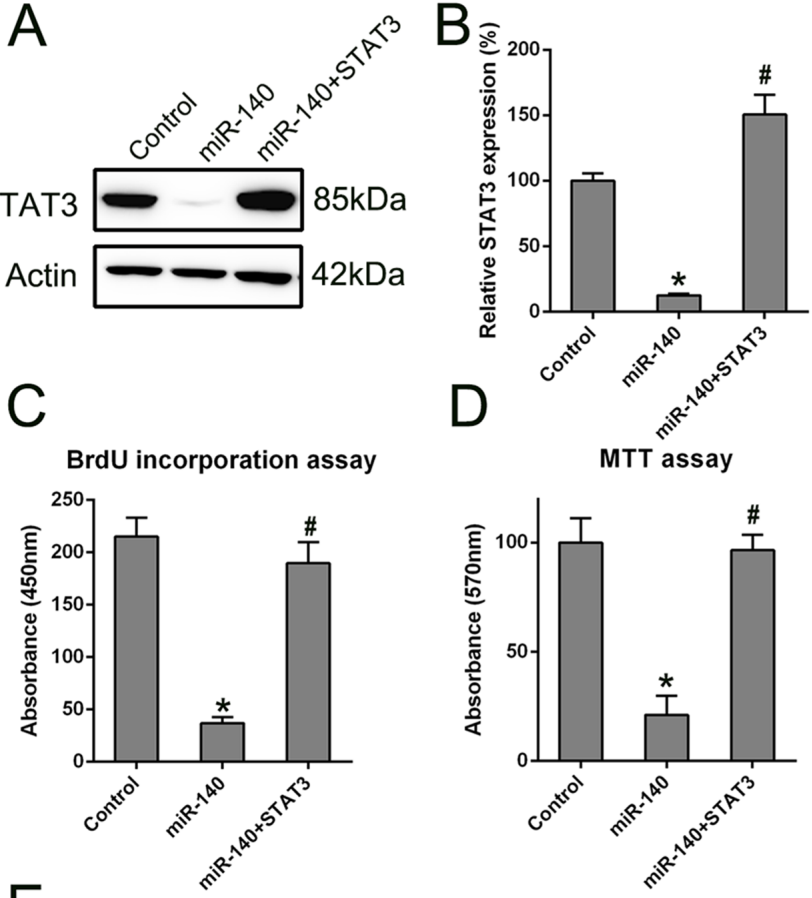

MTT assay
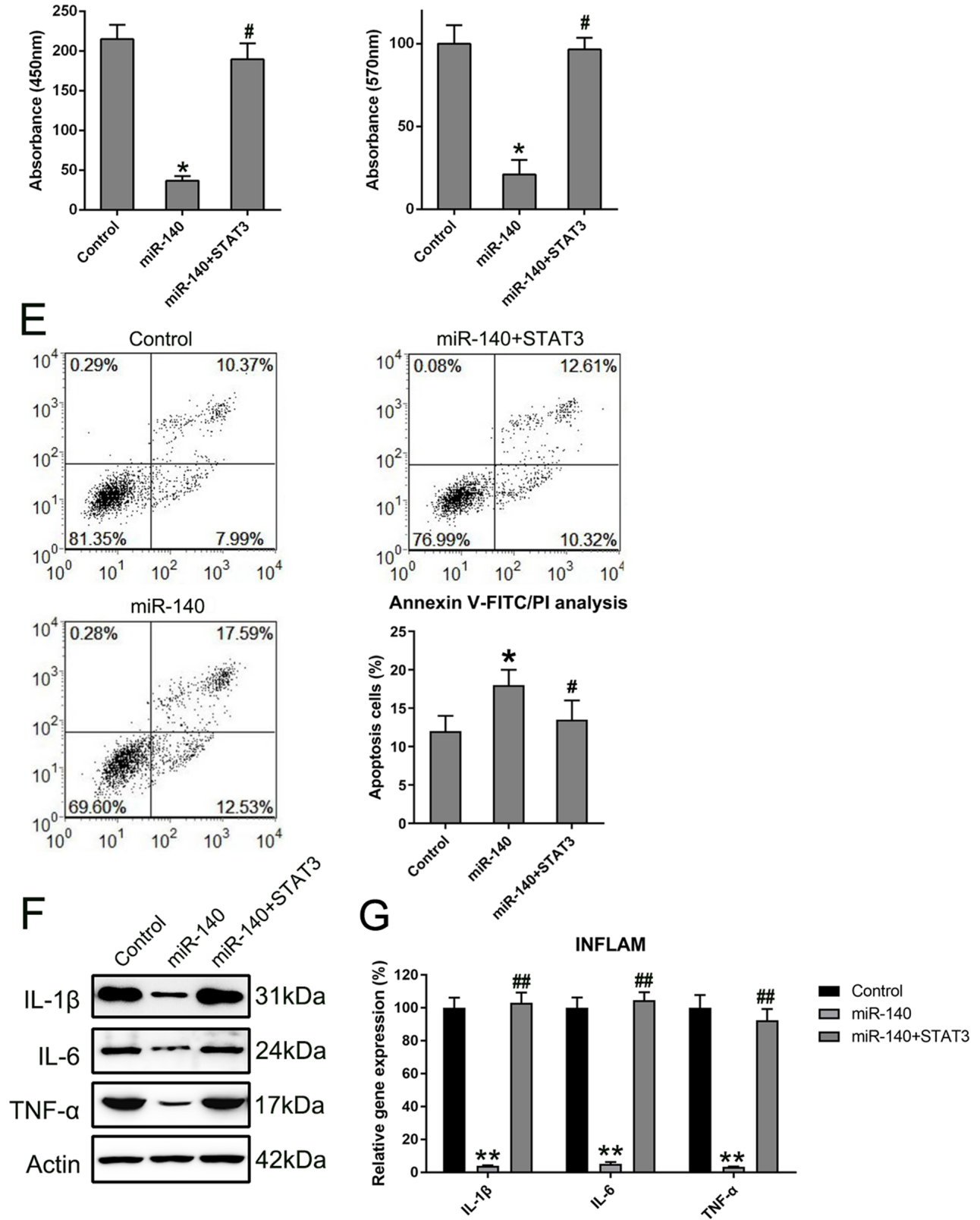

Figure 7. STAT3 restored the regulatory role of miR-140 in RA FLSs. STAT3 expression levels were detected in cells using (A) western blotting and (B) RT-qPCR following miR-140 and STAT3 transfections. All groups were normalized to the control group (100\%). The proliferative ability of cells expressing STAT3 and miR-140, miR-140 alone, or control was measured at $48 \mathrm{~h}$ post-transfection using the (C) BrdU incorporation assays and (D) MTT assays. (E) The apoptotic rate of RA FLSs in each group was determined by annexin V-FITC/PI flow cytometry. Early apoptotic cells and later apoptotic cells are indicated in the top right and bottom right quadrants, respectively, in each plot. Quantitative analysis of the apoptotic rate of RA FLSs in two groups is displayed in the right panel. (F) Western blotting was carried out to assess the expression levels of pro-inflammatory cytokines regulated by the miR-140 and STAT3 plasmid transfections. (G) RT-qPCR was performed to examine the gene expression of cytokines from the RA FLSs after transfection. All groups were normalized to the control group $(100 \%)$. Data represents the mean $\pm \mathrm{SD}$. ${ }^{*} \mathrm{P}<0.05,{ }^{* *} \mathrm{P}<0.01$ vs. the control group; ${ }^{\sharp} \mathrm{P}<0.05,{ }^{\# / \prime} \mathrm{P}<0.01$ vs. the miR-140 group. FLS, fibroblast-like synoviocyte; IL, interleukin; miR, microRNA; PI, propidium iodide; RA, rheumatoid arthritis; RT-qPCR, reverse transcription-quantitative PCR; TNF, tumor necrosis factor. 
inflammatory cytokines in RA FLSs, exerted through STAT3. As such, the interplay between miR-140 and STAT3 may serve as an effective therapeutic target for the treatment of RA in humans.

\section{Acknowledgements}

Not applicable.

\section{Funding}

No funding was received.

\section{Availability of data and materials}

The datasets used and/or analyzed during the current study are available from the corresponding author on reasonable request.

\section{Authors' contributions}

$\mathrm{JZ}$ conceived and designed the research, and interpreted the results of the experiments. JW contributed to the design of the study and the interpretation of experimental results. $\mathrm{JH}$, WD, YH, HP, and JL performed experiments, analyzed data, prepared figures and drafted the manuscript. WD and $\mathrm{YH}$ also approved the final version of manuscript. HP and JL also edited and revised manuscript.

\section{Ethics approval and consent to participate}

All participants gave written informed consent prior to participating and the current study was approved by the Ethics Committee of Zunyi Medical University.

\section{Patient consent for publication}

Not applicable.

\section{Competing interests}

The authors declare that they have no competing interests.

\section{References}

1. Meier FM, Frerix M, Hermann W and Muller-Ladner U: Current immunotherapy in rheumatoid arthritis. Immunotherapy 5: 955-974, 2013.

2. Boissier MC, Semerano L, Challal S, Saidenberg-Kermanac'h N and Falgarone G: Rheumatoid arthritis: From autoimmunity to synovitis and joint destruction. J Autoimmun 39: 222-228, 2012

3. Tobon GJ, Youinou P and Saraux A: The environment, geo-epidemiology, and autoimmune disease: Rheumatoid arthritis. J Autoimmun 35: 10-14, 2010.

4. Rockel JS and Kapoor M: Autophagy: Controlling cell fate in rheumatic diseases. Nat Rev Rheumatol 13: 193, 2017.

5. Bartok B, Hammaker D and Firestein GS: Phosphoinositide 3-kinase delta regulates migration and invasion of synoviocytes in rheumatoid arthritis. J Immunol 192: 2063-2070, 2014.

6. Qin B, Yang M, Fu H, Ma N, Wei T, Tang Q, Hu Z, Liang Y, Yang Z and Zhong R: Body mass index and the risk of rheumatoid arthritis: A systematic review and dose-response meta-analysis. Arthritis Res Ther 17: 86, 2015.

7. Qin B, Liang Y, Yang Z and Zhong R: Response to: 'Body mass index and the risk of rheumatoid arthritis: A systematic review and dose-response meta-analysis'-authors' reply. Arthritis Res Ther 17: 217, 2015
8. Lee RC, Feinbaum RL and Ambros V: The C. Elegans heterochronic gene lin-4 encodes small RNAs with antisense complementarity to lin-14. Cell 75: 843-854, 1993.

9. Kozomara A and Griffiths-Jones S: miRBase: Integrating microRNA annotation and deep-sequencing data. Nucleic Acids Res 39: D152-D157, 2011.

10. Filkova M, Jungel A, Gay RE and Gay S: MicroRNAs in rheumatoid arthritis: Potential role in diagnosis and therapy. BioDrugs 26: 131-141, 2012.

11. Li S, Jin Z and Lu X: MicroRNA-192 suppresses cell proliferation and induces apoptosis in human rheumatoid arthritis fibroblast-like synoviocytes by downregulating caveolin 1 . Mol Cell Biochem 432: 123-130, 2017.

12. Kurowska-Stolarska M, Alivernini S, Melchor EG, Elmesmari A, Tolusso B, Tange C, Petricca L, Gilchrist DS, Di Sante G, Keijzer C, et al: MicroRNA-34a dependent regulation of AXL controls the activation of dendritic cells in inflammatory arthritis. Nat Commun 8: 15877, 2017.

13. Gao W, McCormick J, Connolly M, Balogh E, Veale D and Fearon U: Hypoxia and STAT3 signalling interactions regulate pro-inflammatory pathways in rheumatoid arthritis. Ann Rheum Dis 74: 1275-1283, 2015.

14. Isomaki P, Junttila I, Vidqvist KL, Korpela M and Silvennoinen O: The activity of JAK-STAT pathways in rheumatoid arthritis: constitutive activation of STAT3 correlates with interleukin 6 levels. Rheumatology (Oxford) 54: 1103-1113, 2015.

15. Nowell MA, Richards PJ, Fielding CA, Ognjanovic S, Topley N, Williams AS, Bryant-Greenwood G and Jones SA: Regulation of pre-B cell colony-enhancing factor by STAT-3-dependent interleukin-6 tranes-signaling: Implications in the pathogenesis of rheumatoid arthritis. Arthritis Rheum 54: 2084-2095, 2006.

16. Turkson J and Jove R: STAT proteins: Novel molecular targets for cancer drug discovery. Oncogene 19: 6613-6626, 2000.

17. Turkson J, Bowman T, Garcia R, Caldenhoven E, De Groot RP and Jove R: Stat 3 activation by src induces specific gene regulation and is required for cell transformation. Mol Cell Biol 18: 2545-2552, 1998 .

18. Chen Z and Han ZC: STAT3: A critical transcription activator in angiogenesis. Med Res Rev 28: 185-200, 2008.

19. Devarajan E and Huang S: STAT3 as a central regulator of tumor metastases. Curr Mol Med 9: 626-633, 2009.

20. Starr R, Willson TA, Viney EM, Murray LJ, Rayner JR, Jenkins BJ, Gonda TJ, Alexander WS, Metcalf D, Nicola NA and Hilton DJ: A family of cytokine-inducible inhibitors of signalling. Nature 387: 917-921, 1997.

21. Endo TA, Masuhara M, Yokouchi M, Suzuki R, Sakamoto H, Mitsui K, Matsumoto A, Tanimura S, Ohtsubo M, Misawa H, et al: A new protein containing an $\mathrm{SH} 2$ domain that inhibits JAK kinases. Nature 387: 921-924, 1997.

22. Coombs JH, Bloom BJ, Breedveld FC, Fletcher MP, Gruben D, Kremer JM, Burgos-Vargas R, Wilkinson B, Zerbini CA and Zwillich SH: Improved pain, physical functioning and health status in patients with rheumatoid arthritis treated with CP-690,550, an orally active Janus kinase (JAK) inhibitor: Results from a randomised, double-blind, placebo-controlled trial. Ann Rheum Dis 69: 413-416, 2010.

23. Tanaka Y, Suzuki M, Nakamura H, Toyoizumi S and Zwillich SH; Tofacitinib Study Investigators: Phase II study of tofacitinib (CP-690,550) combined with methotrexate in patients with rheumatoid arthritis and an inadequate response to methotrexate. Arthritis Care Res (Hoboken) 63: 1150-1158, 2011.

24. Dong W, Yao C, Teng X, Chai J, Yang X and Li B: miR-140-3p suppressed cell growth and invasion by downregulating the expression of ATP8A1 in non-small cell lung cancer. Tumour Biol 37: 2973-2985, 2016.

25. Wang Z, Hu J, Pan Y, Shan Y, Jiang L, Qi X and Jia L: miR-140-5p/miR-149 affects chondrocyte proliferation, apoptosis, and autophagy by targeting FUT1 in osteoarthritis. Inflammation 41: 959-971, 2018.

26. Flamini V, Jiang WG and Cui Y: Therapeutic role of miR-140-5p for the treatment of non-small cell lung cancer. Anticancer Res 37: 4319-4327, 2017.

27. Li W and He F: Monocyte to macrophage differentiation-associated (MMD) targeted by miR-140-5p regulates tumor growth in non-small cell lung cancer. Biochem Biophys Res Commun 450: 844-850, 2014.

28. Xie WB, Liang LH, Wu KG, Wang LX, He X, Song C, Wang YQ and Li YH: miR-140 expression regulates cell proliferation and targets PD-L1 in NSCLC. Cell Physiol Biochem 46: 654-663, 2018. 
29. Yuan Y, Shen Y, Xue L and Fan H: miR-140 suppresses tumor growth and metastasis of non-small cell lung cancer by targeting insulin-like growth factor 1 receptor. PLoS One 8: e73604, 2013.

30. Song B, Wang Y, Xi Y, Kudo K, Bruheim S, Botchkina GI, Gavin E, Wan Y, Formentini A, Kornmann M, et al: Mechanism of chemoresistance mediated by miR-140 in human osteosarcoma and colon cancer cells. Oncogene 28: 4065-4074, 2009.

31. Wei R, Cao G, Deng Z, Su J and Cai L: miR-140-5p attenuates chemotherapeutic drug-induced cell death by regulating autophagy through inositol 1,4,5-trisphosphate kinase 2 (IP3k2) in human osteosarcoma cells. Biosci Rep 36: e00392, 2016.

32. Xiao Q, Huang L, Zhang Z, Chen X, Luo J, Zhang Z, Chen S, Shu Y, Han Z and Cao K: Overexpression of miR-140 inhibits proliferation of osteosarcoma cells via suppression of histone deacetylase 4. Oncol Res 25: 267-275, 2017.

33. Li H, Guan SB, Lu Y and Wang F: miR-140-5p inhibits synovial fibroblasts proliferation and inflammatory cytokines secretion through targeting TLR4. Biomed Pharmacother 96: 208-214, 2017.

34. Liu X, Wang S, Yuan A, Yuan X and Liu B: MicroRNA-140 represses glioma growth and metastasis by directly targeting ADAM9. Oncol Rep 36: 2329-2338, 2016.

35. Rothman AM, Arnold ND, Pickworth JA, Iremonger J, Ciuclan L, Allen RM, Guth-Gundel S, Southwood M, Morrell NW, Thomas M, et al: MicroRNA-140-5p and SMURF1 regulate pulmonary arterial hypertension. J Clin Invest 126: 2495-2508, 2016.

36. Zhang R, Ma J and Yao J: Molecular mechanisms of the cartilage-specific microRNA-140 in osteoarthritis. Inflamm Res 62: 871-877, 2013

37. Aggarwal R, Rider LG, Ruperto N, Bayat N, Erman B, Feldman BM, Oddis CV, Amato AA, Chinoy H, Cooper RG, et al: 2016 American college of rheumatology/european league against rheumatism criteria for minimal, moderate, and major clinical response in adult dermatomyositis and polymyositis: An international myositis assessment and clinical studies group/paediatric rheumatology international trials organisation collaborative initiative. Ann Rheum Dis 76: 792-801, 2017.

38. Livak KJ and Schmittgen TD: Analysis of relative gene expression data using real-time quantitative PCR and the 2(-Delta Delta C(T)) method. Methods 25: 402-408, 2001.

39. Agarwal V, Bell GW, Nam JW and Bartel DP: Predicting effective microRNA target sites in mammalian mRNAs. Elife 12: 4, 2015.

40. Friedman RC, Farh KK, Burge CB and Bartel DP: Most mammalian mRNAs are conserved targets of microRNAs. Genome Res 19: 92-105, 2009.

41. Lan H, Chen W, He G and Yang S: miR-140-5p inhibits ovarian cancer growth partially by repression of PDGFRA. Biomed Pharmacother 75: 117-122, 2015.

42. Alsaleh G, Francois A, Philippe L, Gong YZ, Bahram S, Cetin S, Pfeffer S, Gottenberg JE, Wachsmann D, Georgel P and Sibilia J: miR-30a-3p negatively regulates BAFF synthesis in systemic sclerosis and rheumatoid arthritis fibroblasts. PLoS One 9: e111266, 2014

43. Alivernini S, Kurowska-Stolarska M, Tolusso B, Benvenuto R, Elmesmari A, Canestri S, Petricca L, Mangoni A, Fedele AL, Di Mario C, et al: MicroRNA-155 influences B-cell function through PU.1 in rheumatoid arthritis. Nat Commun 7: 12970, 2016 .
44. Chen SY: MicroRNA-223: A double-edged sword in rheumatoid arthritis. Rheumatol Int 34: 285-286, 2014.

45. Shi DL, Shi GR, Xie J, Du XZ and Yang H: MicroRNA-27a inhibits cell migration and invasion of fibroblast-like synoviocytes by targeting follistatin-like protein 1 in rheumatoid arthritis. Mol Cells 39: 611-618, 2016

46. Chen Y, Xian PF, Yang L and Wang SX: MicroRNA-21 promotes proliferation of fibroblast-like synoviocytes through mediation of $\mathrm{NF}-\kappa \mathrm{B}$ nuclear translocation in a rat model of collagen-induced rheumatoid arthritis. Biomed Res Int 2016: 9279078, 2016.

47. Zhang F and Wu Z: Significantly altered expression of miR-511-3p and its target AKT3 has negative prognostic value in human prostate cancer. Biochimie 140: 66-72, 2017.

48. Pais H, Nicolas FE, Soond SM, Swingler TE, Clark IM, Chantry A, Moulton V and Dalmay T: Analyzing mRNA expression identifies Smad3 as a microRNA-140 target regulated only at protein level. RNA 16: 489-494, 2010.

49. Sun J, Shi R, Zhao S, Li X, Lu S, Bu H, Ma X and Su C: E2F8, a direct target of miR-144, promotes papillary thyroid cancer progression via regulating cell cycle. J Exp Clin Cancer Res 36: 40, 2017.

50. Masuda M, Suzui M, Yasumatu R, Nakashima T, Kuratomi Y, Azuma K, Tomita K, Komiyama S and Weinstein IB: Constitutive activation of signal transducers and activators of transcription 3 correlates with cyclin D1 overexpression and may provide a novel prognostic marker in head and neck squamous cell carcinoma. Cancer Res 62: 3351-3355, 2002.

51. Kim BH, Yi EH and Ye SK: Signal transducer and activator of transcription 3 as a therapeutic target for cancer and the tumor microenvironment. Arch Pharm Res 39: 1085-1099, 2016.

52. Cafferkey $\mathrm{C}$ and Chau I: Novel STAT 3 inhibitors for treating gastric cancer. Expert Opin Investig Drugs 25: 1023-1031, 2016.

53. Bosch-Barrera J, Queralt B and Menendez JA: Targeting STAT3 with silibinin to improve cancer therapeutics. Cancer Treat Rev 58: 61-69, 2017.

54. Xiong H, Zhang ZG, Tian XQ, Sun DF, Liang QC, Zhang YJ, Lu R, Chen YX and Fang JY: Inhibition of JAK1, 2/STAT3 signaling induces apoptosis, cell cycle arrest, and reduces tumor cell invasion in colorectal cancer cells. Neoplasia 10: 287-297, 2008.

55. Liu Y, Li PK, Li C and Lin J: Inhibition of STAT3 signaling blocks the anti-apoptotic activity of IL-6 in human liver cancer cells. J Biol Chem 285: 27429-27439, 2010.

56. Aoki Y, Feldman GM and Tosato G: Inhibition of STAT3 signaling induces apoptosis and decreases survivin expression in primary effusion lymphoma. Blood 101: 1535-1542, 2003.

57. Meng Q, Du X, Wang H, Gu H, Zhan J and Zhou Z: Astragalus polysaccharides inhibits cell growth and pro-inflammatory response in IL-1 $\beta$-stimulated fibroblast-like synoviocytes by enhancement of autophagy via PI3K/AKT/mTOR inhibition. Apoptosis 22: 1138-1146, 2017.

58. Li J, Li Y, Jiao J, Wang J, Li Y, Qin D and Li P: Mitofusin 1 is negatively regulated by microRNA 140 in cardiomyocyte apoptosis. Mol Cell Biol 34: 1788-1799, 2014.

(7) $\Theta$ This work is licensed under a Creative Commons Attribution-NonCommercial-NoDerivatives 4.0 International (CC BY-NC-ND 4.0) License. 